Sp. gr. at $20^{\circ}$. Sapon, val.

Oil obtained by extraction of kernels*..... 0.952

Oil obtained by extraction of seeds....... 0.921

Oil obtained by expression of kernels...... $0.95^{8}$
-...

136.9

138

146

147.2
Iod. val. Acid val.

....

$94.6 \quad 42$

... 44

$108 \quad 56$

... 54

The amount of oil obtained by heptane extraction of the kernels was too small to admit of extended analytical study. The difference between both physical and chemical constants of the heptane extract of the comminuted seeds and the expressed kernels can readily be explained by the difference in the two products consequent by the method employed. The heptane extract was not only that of the kernels but that of the seed coats as well. A comparison of the yield of the heptane extract of the seeds with that of the expressed oil from the kernels reveals clearly that the expression was far from quantitative.

The Oleoresin of the Cones.--When the oleoresin of Digger's pine is referred to, that of the wood is generally implied. This has received attention on the part of several investigators. Another oleoresin, quite different in appearance, is that which oozes from the tips of the scales of the cones. It is sulphur-yellow, clear and transparent, when fresh, but upon prolonged exposure it loses its transparency and softness and becomes more or less brittle.

From ten cones $77.6 \mathrm{Gm}$. of oleoresin were collected, averaging $7.6 \mathrm{Gm}$. per cone. Inasmuch as the average weight of a cone is $6 \mathrm{Io} \mathrm{Gm}$., it becomes apparent that slightly more than $\mathrm{I}$ percent of oleoresin was thus obtained. Presumably a larger amount could be obtained by extraction with the proper solvent.

The saponification value was determined according to the U. S. P. (pp. 535 and 536 ), the acid value by the indirect method, according to the same standard (p. 13I).

Sap. No. 152.9 and 154.5 . Acid No. 147.0 and 147.0 .

NoTE.-The distillation of a number of fresh cones several years ago yielded but a few $\mathrm{Cc}$. of a volatile oil which thickened before it could be investigated.

\title{
A NEW DIGESTANT.*
}

BY W. A. KONANTZ.

A great deal of criticism has been brought against many of the National Formulary and commercial galenicals intended as digestants, on the ground that they are unscientific, and can possibly have no value apart from that which may be ascribed to purely psychological influences. It is, indeed, remarkable how little consideration has been given, in formulating these preparations, to the fundamental physiologic facts relating to digestion and digestive ferments, or to the properties and incompatibilities of the substances combined. Of the nineteen digestants in the National Formulary, twelve are absolutely ineligible in the light of these facts, three are of doubtful utility, and the remaining four, while free from other serious objection, are so limited in their scope and so feeble in their power that, from the standpoint of practical therapeutics, they are useful

* Read before Scientific Section, A. Ph. A., Atlantic City meeting, rgr6. 
only as vehicles. Of the commercial preparations, as a whole, it may be said that they are inferior to the official, even as vehicles. It would seem, therefore, that there is need of a new digestant which will be at least compatible within itself and of such strength as to have real therapeutic value.

In order to understand what should, as well as what should not, enter into this class of preparations, and also what they can be reasonably expected to accomplish, one must at the outset take into consideration four all-important things: First, the nature of the materials which are taken into the body as foodstuffs; second, the processes by which the body normally takes care of these materials; third, the pathological conditions which may cause these processes to vary or to cease completely; and fourth, the nature of the substances which can be used to overcome these conditions. In a paper of this length it is obviously impossible to go deeply into the chemistry of foods, or to say much concerning the physiology and pathology of the digestive system, important as a knowledge of these things is for our purpose, and therefore only the more important facts, and such as are necessary to an understanding of what is to be said later, can be touched upon in the paragraphs which follow. We may begin with the different kinds of foodstuffs.

As is well known, the essential constituents of all organic foodstuffs belong to three classes of chemical compounds; namely, the proteins, the carbohydrates, and the fats. Of these the proteins are by far the most important, since they are the only organic foodstuffs which are indispensable to the body, and which can not be replaced by any other nutrient material. They are found in all animal and vegetable tissue, and they are therefore never absent from any animal or vegetable food. The various kinds of proteins which occur in the different animal and vegetable tissues, such as albumen, myosin, casein, gluten, legumen, etc., differ greatly in many of their physical and chemical properties, but they all are alike in containing the same five elements, carbon, hydrogen, oxygen, nitrogen and stilphur; in never occurring in true solution, but in one of two forms, either colloidal or coagulated; and also in a certain agreement in their products of decomposition. It would seem that the different proteins are composed of the same proximate principles combined in varying proportions. The change from the colloidal to the coagulated form may be brought about by heat, varying with the different proteids from $55^{\circ}$ to $57^{\circ} \mathrm{C}$., and in some cases by alcohol or mineral acids. On decomposition by the putrefactive organisms, the proteins yield a large number of substances, among which are hydrogen sulphide, ammonia, ptomaines, and various amino acids; on hydrolysis with chemical reagents or the digestive ferments, they give first various highly complex products, called albumoses, proteoses, and peptones, and finally a great number of simpler compounds such as the amino acids. These decompositions are of the greatest importance in digestion, since by means of them alone can the huge protein molecule be rendered soluble, diffusible, and assimilable by the body. It will be seen that the digestion of the proteins is of prime importance to the body, of more importance indeed than that of the carbohydrates and fats, which we must consider next.

The carbohydrates and the fats offer a contrast to the proteins in being free from nitrogen and sulphur, both containing the same three elements, carbon, hydrogen, and oxygen, although their quantitative composition is quite differ- 
ent, the fats being much poorer in oxygen, and richer in carbon and hydrogen. They are not so essential to life as are the proteins, and the problem of their digestion is correspondingly less important. They do not seem to be capable of replacing the exhausted constituents of the body, and their main service is undoubtedly in furnishing the body with heat and energy.

The most important carbohydrates, so far as their use in the body is concerned, are the starches, the gums, and the sugars, which differ greatly in their physical properties but have a close chemical relation as is indicated by the nature of their decomposition products. On hydrolysis, all the higher carbohydrates are ultimately converted into the lower ones, the simple sugars, which alone are assimilable by the body. On putrefaction or fermentation, they undergo various decompositions, depending on the nature of the organism present-thus we have the lactic acid fermentation, butyric acid fermentation, alcoholic fermentation, etc. Decompositions of the first kind take place during the normal processes of digestion, while those of the second kind frequently occur during abnormal conditions, and give rise to some common pathological disturbances.

Compared with the proteins and carbohydrates, the fats are simple bodies; they are mostly mixtures of the esters of certain higher fatty acids with glycerin, and they readily decompose into these constituent parts when hydrolyzed. When the hydrolysis is effected by alkalies, the fatty acids which are freed combine with the bases to form soaps, and these soaps have the power to emulsify large quantities of the same or other fat. This fact is of great importance in digestion, since it. appears that the fats can be absorbed into the body only after conversion into such soaps or emulsified fats.

From what has already been said, it can be readily seen that nearly all the organic foodstuffs are insoluble in water. Fats, as we all know, are not miscible with water, the proteins only swell without actually dissolving in it, and, of the carbohydrates, the sugars alone are soluble. On being introduced into the alimentary canal, the foodstuffs, although surrounded by the body, are still outside of what is called the body proper. To get from the digestive tract into the body proper, the foodstuffs must pass through the mucous membrane lining of these organs and also the walls of blood or lymph vessels. Only liquids can make this passage, so it is necessary to reduce to the liquid state all foodstuffs not already in that condition. This reduction to the liquid state constitutes the digestive processes, and is effected in the body by five secretions; namely, the salivary juice, the gastric juice, the pancreatic juice, the intestinal juice, and the bile. In this process water is the solvent, and certain chemical agents convert the insoluble nutrients into substances that are soluble in water.

The first of these secretions to act upon the foodstuffs is the salivary juice, a transparent and somewhat slimy liquid of slightly alkaline reaction. It consists chiefly of water (about 99 percent), holding in solution certain salts, mucin, and an enzyme called ptyalin, which has the power to convert starch into dextrin and sugar. Approximately $\mathrm{r} 500 \mathrm{Cc}$. of saliva are normally secreted in the course of 24 hours, and this secretion might therefore be expected to play an important part in the chemical processes of digestion, but it has not yet been found that it does so. The saliva has no effect on most articles of diet; starch alone is converted into dextrin and sugar, and even this action is very inconsiderable, almost noth- 
ing when compared with the similar action of the pancreatic juice or of diastase. This is because the period during which the ptyalin can act is of very short duration. Its action on starch takes place only in the faintly alkaline reaction which belongs to the normal saliva, and its action is immediately enfeebled or entirely neutralized when it reaches the acid gastric juice. Thus only a small portion of the starch consumed is split up by the salivary juice. The chief value of the salivary juice is undoubtedly in the amount of water which it furnishes, and which is utilized, first, to lubricate the foods and prevent their irritation of the mucous membranes, and secondly, as the digestive solvent for the foods as they become soluble.

The next secretion to act upon the foodstuffs is the gastric juice, and with it begins the definite work of dissolving those foods which are insoluble in water. It is distinguished from all the other digestive fluids by its acid reaction, which is due to the presence of $0.2-0.4$ percent of hydrochloric acid. In addition to this it contains certain salts and three enzymes, pepsin, rennin, and a lipase. The chief substances digested by the gastric juice are the proteins, which are converted by the pepsin into proteoses and peptones-substances which, unlike the proteins, are neither colloidal nor coagulable, are more easily diffusible through animal membranes, and therefore better suited for absorption into the blood. This action of pepsin can occur only in an acid medium, a condition which is supplied by the hydrochloric acid. The characteristic action of rennin is the coagulation of colloidal protein, like the albumen of milk, which is then acted upon by the pepsin and hydrochloric acid in the same manner as the other proteins. The protein membranes which surround the fat cells of certain foods are also dissolved by the pepsin, setting free the fat, which is attacked by the lipase, and split into its constituent fatty acids and glycerin. How large a portion of the fat, however, is thus broken up in the stomach cannot be stated, but it is probably a very small amount, for the decomposition of fats, at least in experiments on artificial digestion, goes on very slowly. Nevertheless, as will be shown later, it is quite sufficient if only a minute part of the fats is thus split up.

We have just said that hydrochloric acid is necessary for the action of pepsin, but this is not its only significance. Recent researches indicate that one of its. important functions is the killing of microörganisms which reach the stomach with the food, and which would otherwise set up processes of decomposition in the alimentary cana1, and thus destroy a part of the food before its absorption, while the products of decomposition would produce disagreeable symptoms and even disease. The mineral acids have antiseptic power even in such dilution as. that of the hydrochloric acid of the gastric juice. Boer found that hydrochloric acid in the proportion of 0.15 percent effectually restrained the growth of all bacteria with which he experimented, and that most forms were impeded by as small amounts as one part in two thousand of this acid. Besides these functions, it has been fairly well established that cane sugar and maltose (double sugars) are converted by the hydrochloric acid into glucose and levulose (simple sugars); that the muscular activity of the stomach, so important in mechanically disintegrating the foodstuffs and mixing them with the digestive juice, is largely governed by the relative acidity on the two sides of the pylorus; and that the stimulation of pancreatic secretion, with its important digestive ferments, is a hormone formed 
by the action of the hydrochloric acid on duodenal epithelium. In the opinion of the writer, the treatment which the foodstuffs receive in the gastric juice is the most important to which they are subjected, for if the stomach fails to produce in its contents the general characteristics of chyme there will be driven out into the duodenum a mass of semi-digested or fermenting material unfit for intestinal digestion, thereby disordering the functions of these parts and all the others which follow.

The pancreatic juice, by which the foods are next acted upon, is a sort of "finisher." It acts with vigor upon all the three main groups of foodstuffs, completing the reactions begun by the salivary and gastric juices. It converts the starch into maltose, completing the work begun by the saliva. This action is due to the presence of an enzyme, amylopsin, which is similar to ptyalin but is more vigorous. It changes proteins into proteoses and peptones, completing the work begun by the gastric juice. This is accomplished by another enzyme, trypsin, which is similar to, but more active, than pepsin. It decomposes the fats into their constituent parts, and in this work the active agent is the enzyme steapsin. There is also present an enzyme similar to the rennin of the gastric juice. Perhaps the most notable feature of the pancreatic juice is its alkaline reaction, which is due to sodium carbonate. The sodium carbonate has three functions: first, it furnishes the alkaline medium which is necessary for the action of the pancreatic enzymes just mentioned; second, it neutralizes the hydrochloric acid of the gastric juice; and third, it neutralizes the fatty acids set free from the fats by the lipase and the steapsin, and thereby forms soaps, which serve to emulsify the rest of the fats by surrounding their globules with such a coating that they are unable to unite into large masses, and become thereby capable of being absorbed through the walls of the intestines. It was said in a preceding paragraph that only a small quantity of the fats is probably split up into free fatty acids and glycerin by the lipase or the steapsin, and that this small quantity is quite sufficient. This is true because the whole amount of fat is thereby rendered capable of being converted into a fine emulsion, in which form it is assimilable. It must not be forgotten that some free fatty acid, however small in amount, is necessary for this saponification with sodium carbonate, since neutral fats can be saponified only by free alkalies. The carbonates of the alkalies have no action on the neutral fats but only on the free fatty acids, and the sodium carbonate is therefore unable to decompose the fat-molecule, or to form a soap, until some fatty acid has been set free by the ferments, nor will it emulsify the fat if no fatty acid is present, but a fine emulsion is produced after the decomposition. The presence of free fatty acid and sodium carbonate is therefore the most essential condition in the digestion of the fats.

The remaining two digestive fluids, the intestinal juice and the bile, seem to act chiefly as aids to the pancreatic juice; neither contains any enzymes of importance in digestion. The chief constituent of the intestinal juice is sodium carbonate, and its function is probably to aid in neutralizing the hydrochloric acid and in emulsifying the fats. The bile also plays an important rôle in the digestion and absorption of fats by acting as a solvent of the fatty acids and soaps, and therefore as a carrier of them into the mucous membranes.

Having now considered the normal processes of digestion, it is equally important for our purpose to know something of the pathological conditions which 
may cause these processes to vary or to cease, for digestants are used, of course, not when the functions are normal, but when they are abnormal. With regard to cause, we may say that digestive disturbances are of two kinds: those which have their causes directly in the alimentary tract, and those which have their causes in other parts of the body, the digestive system simply being involved in a more or less general decline of bodily functions. Disturbances of the first kind are due chiefly to a neglect of hygienic measures, as the eating of improper foods or improperly prepared foods, irregularity in eating, overeating, incomplete mastication, and so on; but they may be due to acute or chronic gastritis, or even to organic changes in the abdominal viscera, as in carcinoma and ulcer. The second kind of disturbances occur during short or prolonged fevers, during infectious diseases, in chronic maladies, as anemia, chlorosis, diabetes, tuberculosis, and in fact whenever the system in general is involved; they are due to the impoverishment of the blood, and in consequence the secretions of the glands are diminished, and the motility of the muscles concerned with peristalsis enfeebled. In either case we have much the same conditions to deal with: deficient secretion of digestive enzymes; hypoacidity, or deficient acidity of the gastric juice; hyperacidity, or excessive acidity of the gastric juice (which forms one variety of acid stomach); atony of the muscles of peristalsis; ferment action, or rather putrefaction, of the foods, with formation of lactic acid, butyric acid, and other effete products; flatulence, caused by this fermentation and the liberation of gas; hypersecretion of mucus; and irritation of the walls of the alimentary canal, due to the presence of undigested or indigestible food.

It should be noted that digestive disturbances are nearly always symptoms, not diseases; their treatment must therefore be chiefly symptomatic. However, to secure any permanent relief the underlying causes must of course be removed. If they are due to neglect of hygienic measures, then a strict diet must be instituted and rigidly enforced; if they are due to diseases then these diseases must be given treatment. In any case, the writer believes that the proper use of a digestant will always be as an adjunct--but a necessary adjunct-- to some other treatment calculated to remove the causes of the digestive failure, and that the sole objects of a digestant are to replace temporarily, digestive ferments that are lacking, to render the alimentary tract aseptic, to counteract disagreeable symptoms, and so far as possible to restore normal conditions in the digestive system. If a digestant does these things, it has done all that can reasonably be expected of it.

'The substances which are most widely used for the accomplishment of these objects are the following, which have been listed according to their chief action:

Digestive Ferments: Pepsin, pancreatin, diastase.

Acids: Hydrochloric, phosphoric, lactic.

Antacids: Sodium bicarbonate, magnesia, lime-water.

Bitters: Strychnine, quinine, gentian, quassia.

Carminatives: Volatile oils, spices, asafœtida.

Antiseptics, (a) gastric: Creosote, resorcinol, sodium phenolsulphonate;

(b) intestinal: betanaphthol, salol, aspirin.

Protectives: Insoluble bismuth salts, wood charcoal.

Demulcents: Mucilages (acacia, elm, linseed, althæa, etc.), glycerin.

Of these substances, the most useful, and the one most likely to do good, is 
undoubtedly hydrochloric acid. The numerous functions which it performs in the normal processes of digestion have already been mentioned. In hypoacidity it is invaluable for supplying the acid which is so necessary for the activation of the peptic glands, for the stimulation of the gastro-intestinal muscles, and for the prevention of fermentation. 'In hyperacidity the excessive secretion may be prevented by its administration, on the rule that acids check acid secretions; or, if the excessive acidity is due to the formation of butyric, lactic and other organic acids from the foods by fermentation, it will by reason of its powerful antiseptic influence effectually overcome this condition. As an antiseptic, hydrochloric acid is decidedly superior to the phenolic compounds which are so often given for this purpose, and which tend to derange the system by inhibiting its ferments. It should be noted here that the method of treating hyperacidity of the second type, based upon the administration of hydrochloric acid, creosote, or other antiseptics, is not for the purpose of removing the acids, but to stop fermentation. The objection to the method of treatment with alkalies, like sodium bicarbonate and magnesia, is that, while they neutralize the acids, they do not prevent the fermentation, and if given during the process of digestion they interfere with it, by neutralizing also the normal acidity of the stomach. Medical writers, on the whole, are agreed that most cases of digestive failure, from whatever causes, are benefited by the administration of hydrochloric acid, perhaps the only exception being where there is much gastric inflammation, and even here it is extremely doubtful whether in the quantity and dilution in which it is ordinarily given hydrochloric acid is at all irritant.

Certain other acids, such as phosphoric and lactic, are occasionally substituted for hydrochloric acid, but without sufficient reason. They certainly cannot perform the functions of the hydrochloric acid in digestion. Phosphoric acid is a normal constituent of the gastric juice in the form of acid phosphates; lactic acid is found only when there is putrefaction of the carbohydrates, and is extremely irritant to mucous membranes. It is therefore the more objectionable of the two.

In those cases of digestive failure which are due to a lack of digestive ferments, practically the only available remedies are pepsin, pancreatin, and diastase. These are not the pure isolated enzymes, but more or less impure mixtures of several enzymes with other albuminous bodies. Pepsin is usually made by the autodigestion of the mucous membrane of the hog's stomach, and the separation of the pepsin by the process of salting out. The United States Pharmacopoeia requires that standard pepsin shall be able to digest not less than three thousand times its own weight of coagulated egg albumin in two and one-half hours at a temperature of $52^{\circ} \mathrm{C}$., but much stronger pepsins are prepared commercially. Pepsin regularly contains some rennin, and its solution will therefore coagulate milk. It has no action on the fats or carbohydrates. Pancreatin is made from the pancreas of the hog in much the same way that pepsin is extracted from the stomach; it is essentially a mixture of the specific ferments found in the pancreatic juice. The United States Pharmacopoeia requires that standard pancreatin shall be able to digest at least twenty-five times its own weight of boiled starch in five minutes at a temperature of $40.5^{\circ} \mathrm{C}$. Pancreatin should also act on the proteins and fats. Diastase is obtained from infusions of malt (barley, oats, wheat, potato, etc.) by precipitation with alcohol; one part should convert two 
thousand parts of starch into dextrin and maltose. It also contains a second ferment, known as peptase, which is capable of changing proteins into peptones.

It will be seen that these preparations are highly powerful substances, and it might be expected that they would be of great value in replacing digestive ferments lacking in the human body, but their utility is greatly lessened by the fact that they are delicate bodies whose activity is destroyed by the presence of many other substances. Thus, the digestive power of pepsin is destroyed or impaired by strong acids (over 0.5 percent), by alkalies, by alcohol (over ro percent), by the soluble salts of many heavy metals, and by gallic or tannic acid, while pancreatin is rendered inert by more than traces of acids or alkalies. In solution these two are mutually incompatible, the pepsin destroying the pancreatin if the solution be acid and the pancreatin destroying the pepsin if the solution be neutral or alkaline. Diastase, on the other hand, will act in either a neutral or a feebly acid solution, although the latter is preferable. At one time it was thought that lactic acid increased the activity of diastase, but it has recently been shown that it has a retarding effect; however, the acid phosphates seem to exert a beneficial influence.

The problem in the case of digestive ferments is chiefly whether to administer pepsin (and hydrochloric acid) or pancreatin (and sodium bicarbonate). Both kinds of solution are largely used, as well as some which are supposed to contain both ferments. These latter are impossible combinations for the reasons already given. The examination of several such preparations by the chemists of the Council of Chemistry and Pharmacy of the American Medical Association has shown them to be inert, or to have only a weak action of the fermenc that happens to be in excess. With regard to the use of pancreatin, it must be borne in mind that it is not active in the presence of free acid, and in the normal gastric juice is not only incapable of affecting digestion but will itself be digested. If used, it must therefore be given enterically, which is inconvenient, or combined with sodium bicarbonate, which is objectionable because the normal acidity of the stomach will be neutralized and digestion retarded instead of promoted. Moreover, the value of pancreatin as a digestant of proteins and fats is practically nil, while its amyloptic power; only $\mathrm{I}$ to 25 , is exceedingly low. It is therefore greatly inferior to pepsin and diastase, which have also the advantage of acting in feebly acid solutions. The most important use of pancreatin is perhaps in making the so-called "predigested" or "peptonized" foods. With milk it is superior to pepsin for this purpose, since the rennin in the latter causes coagulation. As an internal digestant, however, pancreatin has little value, and a combination of pepsin and diastase is much more likely to do good.

Some medical writers contend that pepsin, pancreatin, and diastase are superfluous remedies, because, as they say, the digestive glands rarely fail to secrete their specific ferments. An analogous argument would be that serums are superfluous remedies because the body is capable of forming its own antitoxins. In both cases, the object is to relieve the body, already weakened, from the necessity of manufacturing these agents, to introduce them into the system without delay, and to fortify those that may naturally be present. The statement that the continued use of artificial digestants establishes a habit and a consequent weakening of the natural power to secrete digestive ferments is without foundation; on the other hand, there is evidence that they act as stimulants to the secretory 
glands and promote their functional activity. Pepsin, pancreatin and diastase do good by increasing the number of digestive ferments, thereby increasing the rate and completeness of digestion. This is in accordance with the law of proportionality, which states that when the amount of substance to be acted upon is large in proportion to the quantity of enzyme, then the amount of the reaction is proportionate to the quantity of enzyme present. For example, if we take two solutions of $300 \mathrm{Cc}$. each, containing 3 percent of boiled-starch solution, and add to one $0.5 \mathrm{Cc}$. of malt extract and to the other $2 \mathrm{Cc}$. of malt extract, the greater amount of reaction will take place in the solution to which the larger quantity of malt extract was added. In like manner, the introduction of additional enzymes into the digestive system will increase the rate and completeness of digestion and relieve the system of its load. The artificial ferments are also good demulcents, capable of exerting a soothing influence upon irritated mucous membranes.

For the purpose of overcoming gastro-intestinal irritation the mucilages are the most effective; they are unsuited, however, to incorporation into digestant mixtures. They are usually given in acute cases only, and then in large doses. Certain insoluble substances are also frequently used, like wood-charcoal and the salts of bismuth. These, being heavy and insoluble, gradually spread themselves over the gastro-intestinal mucous membrane, and mechanically protect it against the action of irritant substances in much the same way that paint protects a house against the effects of the weather. The objection to these substances is that they interfere to some extent with digestion, and if used over a considerable period of time, are likely to form concretions or balls which plug the ileo-caecal valve, and otherwise give trouble. For continued use, in combination with other substances, glycerin and syrup are perhaps the most satisfactory.

Those substances which are commonly called "bitters" and which are used to stimulate the appetite, would seem to be contraindicated in digestants because of the possibility that they may cause active gastro-intestinal irritation, or aggravate such a condition if already present. In those cases which are characterized by loss of appetite without special disturbance of the digestive apparatus or in general tonics, they are undoubtedly of value, but when the digestive system is already loaded with more food than it can properly handle, or is in a depressed condition, it is certainly not advisable to increase the load by establishing a false appetite. Perhaps the least irritant of the bitters is strychnine, and it has a decided advantage over the other bitters in its effect upon the muscular movements of the stomach and intestines because it stimulates peristalsis. It is therefore especially valuable in atonic conditions.

Closely allied to the bitters are the so-called aromatics, which depend for their activity upon the presence of a volatile oil. They differ from the bitters in being more powerful but less permanent in their effects, and in exerting their influence in the intestinal canal as well as in the stomach. They are largely used to expel flatus by exciting intestinal peristalsis, for which purpose they are unexcelled. They are also especially useful in disguising the taste of bitter substances, and in covering many other unpleasant flavors. If used in too large doses they are capable of setting up local irritation.

The principal facts relating to digestants have now been given, and upon these 
facts the writer has based the formula of a new digestant, which it is thought avoids the incompatibilities to be found in most other preparations of this kind without sacrificing scope or strength. The proposed formula and directions are as follows:

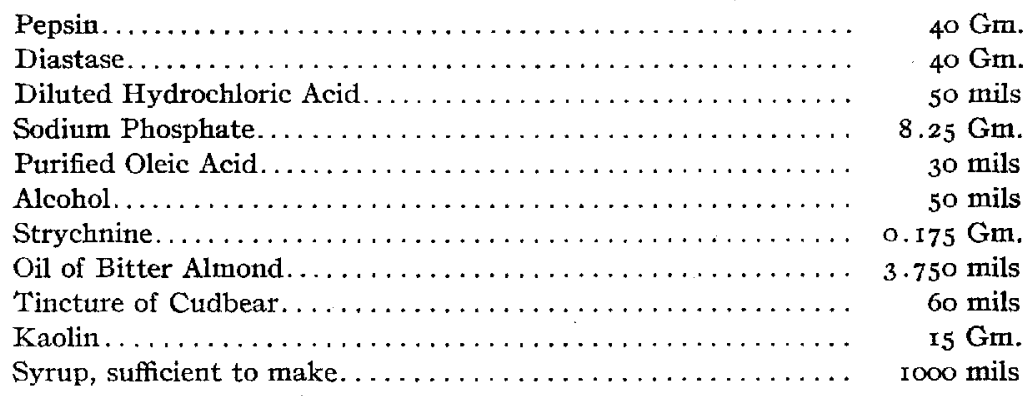

Dissolve the sodium phosphate in the diluted hydrochloric acid, and mix the solution with 725 mils of syrup. In this dissolve the pepsin and diastase, triturate with the kaolin, and filter. Then dissolve the strychnine sulphate in about 25 mils of syrup, and the purified oleic acid and oil of bitter almond in the alcohol. Add these solutions to the filtrate from the first solution. Finally, add the tincture of cudbear, filter, and add syrup sufficient to make 1000 mils.

The use of oleic acid and sodium phosphate in digestant mixtures is new and needs explanation. In the paragraphs on the fats it was shown that free fatty acid is essential to the digestion of the fats. If we take a little neutral fat or oil (that is, one which contains no free fatty acid) and shake it with a solution of sodium carbonate no emulsion will result, but if we now add a drop of a fatty acid like oleic acid, and shake again a fine emulsion forms at once. This is due to the formation of a soap through the interaction of the sodium carbonate with the free fatty acid, and this soap acts as the emulsifying agent. A similar process of emulsification occurs naturally in the digestion of the fats, and is likewise dependent on the presence of free fatty acids. The necessary fatty acid may be liberated from the fats by the organisms of putrefaction or by the lipases of the digestive tract, or it may be added as is done in this preparation. As soon as the fats and fatty acids reach the alkaline intestinal juice the latter will be converted into soap, and the former into an emulsion. It seems reasonable to believe that free fatty acids, administered in small quantities, will aid the digestion of the fats, and it is upon this conviction that the writer has incorporated oleic acid in the above formula.

The sodium phosphate has been added chiefly for two reasons: The first is that diastase seems to act best in a medium containing acid phosphates. The second is that sodium phosphate can combine with free fatty acids to form soaps. It therefore acts in this preparation as an auxiliary to the diastase and to some extent as a substitute for the sodium carbonate of the intestinal canal.

The presence of alcohol in this preparation may seem objectionable to some. The alcoholic content is, however, only about 5 percent by volume, and this amount is necessary to hold the oleic acid and oil of bitter almond in solution. The writer does not believe that this amount is really objectionable, since it has been conclusively shown by numerous investigators that alcohol in amounts less 
than Io percent has no deleterious effect on the processes of digestion. On the contrary, small amounts of alcohol seem to stimulate the secretion of the salivary and gastric glands, and perhaps of the digestive ferments in the intestines. However, the small amount of alcohol which will be taken in each dose of this preparation may be regarded as negligible.

The conicentration of the hydrochloric acid has been fixed at 0.5 percent. This is perhaps the maximum amount that can be used without injury to the pepsin and diastase, and which still gives a fair dose of the acid. Strychnine sulphate has been added for its well-known tonic effects on muscles and glands. The oil of bitter almond has been chosen as the flavor, for it seems to cover the acid taste best. Kaolin is indicated as the clarifying agent, since digestants made with it seem for some reason to be more permanent than those made with talc.

The dose of this preparation is intended to be for an adult two fluidrachms. In such a dose the patient will receive five grains each of pepsin and diastase, six and a half minims of diluted hydrochloric acid, four minims of oleic acid, one grain of sodium phosphate, and one one-hundredth of a grain of strychnine sulphate. This dosage has been arrived at after considerable study, and it is believed that it will give the maximum of therapentic value. If thought too large it can be easily reduced by giving one fluidrachm, or even less. Some writers, however, recommend even much larger doses-thirty grains each of pepsin and diastase, and thirty minims of diluted hydrochloric acid-but there are cases on record where such doses, taken over a considerable period of time, have resulted in serious conditions.

In manufacturing this preparation, only ingredients of known strength should be used, and if there is any doubt they should be standardized before they are used. In this way alone can a uniform and efficient preparation be obtained. Pepsin and diastase having a power, respectively, of $I: 3000$ and $I: 2000$ are best. The diluted hydrochloric acid must be exactly to percent by weight. Purified oleic acid should be used, not the oleic acid of the Pharmacopoeia, as the latter is not intended for internal use, and has an unpleasant odor and taste. The purified oleic acid should be odorless and tasteless. In order to prevent any possible oxidation of the oleic acid, and also to protect the other ingredients of the mixture, it is recommended that the bottles be sealed - this can be easily done by dipping in melted paraffin. The finished preparation should be a perfectly clear solution of a cherry-red color and a cherry flavor.

\section{GLYCERIN AS AN ANTISEP'TIC.}

Glycerin has recently been proved to be a most admirable sterilizing agent, particularly suited for rendering surgical instruments absolutely aseptic. Tests have been made with the bacilli of tuberculosis. When heated in glycerin at a temperature of $120^{\circ} \mathrm{C}$., these germs are invariably killed at the end of one minute. The germs that are killed in this way are those of diphtheria, anthrax, and chicken cholera, as well as Bacillus coli, B. paratyphosus, B. pyocyaneus, B. subtilis, Staphylococcus albus, and Streptococcus brevis. Surgical instruments are uninjured by the treatment, while rubber tubes remain not only uninjured, but are even restored to elasticity when they have become somewhat brittle.-Brit. Journ. Dent. Surg., November 15, 1916, 686; through Pharmaceutical Journal. 